\title{
Reorganization of metastamiRs in the evolution of metastatic aggressive neuroblastoma cells
}

\author{
Faizan H Khan ${ }^{1}$, Vijayabaskar Pandian', Satishkumar Ramraj', Sheeja Aravindan², Terence S Herman ${ }^{1,2}$ \\ and Natarajan Aravindan ${ }^{1 *}$
}

\begin{abstract}
Background: MetastamiRs have momentous clinical relevance and have been correlated with disease progression in many tumors. In this study, we identified neuroblastoma metastamiRs exploiting unique mouse models of favorable and high-risk metastatic human neuroblastoma. Further, we related their deregulation to the modulation of target proteins and established their association with clinical outcomes.

Results: Whole genome miRNA microarray analysis identified 74 metastamiRs across the manifold of metastatic tumors. RT-qPCR on select miRNAs validated profile expression. Results from bio-informatics across the ingenuity pathway, miRCancer, and literature data-mining endorsed the expression of these miRNAs in multiple tumor systems and showed their role in metastasis, identifying them as metastamiRs. Immunoblotting and TMA-IHC analyses revealed alterations in the expression/phosphorylation of metastamiRs' targets, including ADAMTS-1, AKT1/2/3, ASK1, AURK $\beta$, Birc1, Birc2, Bric5, ß-CATENIN, CASP8, CD54, CDK4, CREB, CTGF, CXCR4, CYCLIN-D1, EGFR, ELK1, ESR1, CFOS, FOSB, FRA, GRB10, GSK3ß, IL1a, JUND, KRAS, KRTAP1, MCP1, MEGF10, MMP2, MMP3, MMP9, MMP10, MTA2, MYB, CMYC, NF2, NOS3, P21, pP38, PTPN3, CLEAVED PARP, PKC, SDF-1 $\beta$, SEMA3D, SELE, STAT3, TLR3, TNFa, TNFR1, and VEGF in aggressive cells ex vivo and in a manifold of metastatic tumors in vivo. miRNA mimic (hsa-miR-125b, hsa-miR-27b, hsa-miR-93, hsa-miR-20a) and inhibitor (hsa-miR-1224-3p, hsa-miR-1260) approach for select miRNAs revealed the direct influence of the altered metastamiRs in the regulation of identified protein targets. Clinical outcome association analysis with the validated metastamiRs' targets corresponded strongly with poor overall and relapse-free survival.

Conclusions: For the first time, these results identified a comprehensive list of neuroblastoma metastamiRs, related their deregulation to altered expression of protein targets, and established their association with poor clinical outcomes. The identified set of distinctive neuroblastoma metastamiRs could serve as potential candidates for diagnostic markers for the switch from favorable to high-risk metastatic disease.
\end{abstract}

Keywords: High-risk metastatic disease, Neuroblastoma, MetastamiRS, miRNA, Tumor progression, SH-SY5Y, Aggressive metastatic cells

\section{Background}

Neuroblastoma (NB), the most common cancer at infancy $[1,2]$ accounts for about one tenth of pediatric cancer deaths [3-5]. Despite significant advances in understanding the biology of NB and, improved clinical outcomes in the last decade $[3,5]$, the outcomes for high-risk groups still

\footnotetext{
* Correspondence: naravind@ouhsc.edu

'Department of Radiation Oncology, University of Oklahoma Health Sciences Center, 940, Stanton L. Young Boulevard, BMSB 737, Oklahoma City, OK 73104, USA

Full list of author information is available at the end of the article
}

soar. To that note, cure after relapse of high-risk disease with remarkable heterogeneity, resistance and, poor hematological reserve is extremely rare. Almost half of patients with high-risk NB will relapse with hematogenous metastasis [6] despite intensive multimodal therapy [3, 5, 7-14]. Compared with those with low/intermediate-risk disease (65\%), the five-year overall survival (OS) of patients with high-risk disease is low $(<10 \%)$. The rate of long-term survival is even more dismal ten years after diagnosis, with only $2 \%$ OS for patients with stage 4 disease [13, 15]. Cancer cells should successfully complete multiple

\section{() Biomed Central}


sequential steps, including spreading from the tumor of origin, intravasation, extravasation and colonization, before they will grow and proliferate at a secondary site and form a new tumor $[16,17]$. The capability of cancer cells to metastasize depends on genetic and epigenetic events that are acquired during tumor progression [18]. Despite great advancements in our knowledge of metastasis biology, the molecular mechanisms are still incompletely understood. Remarkably, the regulatory role for miRNAs in metastasis has been established [19-23]. Thus, these miRNAs have been identified as metastamiRs [24], as they have both pro- and anti-metastatic effects. Accordingly, we used a unique, MYCN non-amplified mouse model of human high-risk aggressive metastatic neuroblastoma coupled with whole genome miRNA approach to investigate the functional reorganization of metastamiRs in NB progression.

Genomic amplification of MYCN plays a dominant role in determining the biologic behavior of neuroblastoma and is strongly associated with advanced stage of the disease, rapid tumor progression, therapy resistance and overall poor prognosis [25-29]. However, the realm of MYCN amplification is restricted to about $20 \%$ of all cases of neuroblastoma [26, 30,31], 30-40\% of stage 3 and stage 4 and only $\sim 10 \%$ of stage $4 \mathrm{~s}$ patients $[29,32]$. Critically, the lack of MYCN amplification without either $1 p$ loss or $17 q$ gain further limits the role of MYCN in distinguishing those patients who are likely to survive from those that are destined to fail treatment [33]. Moreover, long-term survival of advanced neuroblastoma patients with MYCN amplification has also been reported [34]. Conversely, prognostic insights and molecular drivers of the MYCN nonamplified high-risk neuroblastoma, that comprises about $60-70 \%$ of stage 3 and stage 4 disease remains unexplored. More importantly, MYCN expression does not correlate with the prognosis of adverse outcome in advanced-stage neuroblastoma with non-amplified MYCN [35]. In this regard, we utilized, MYCN non-amplified cell-line (SH-SY5Y) derived mouse model of human high-risk aggressive metastatic neuroblastoma to define the functional reorganization of metastamiRs in neuroblastoma progression.

miRNAs are endogenous, hairpin-shaped, small noncoding single-stranded RNAs of $\sim 22$ nucleotides in length; they serve as post-transcriptional regulators of gene expression [36]. Although miRNAs were initially considered nonfunctional, recent studies documented the potential of miRNAs to control cell fate [37-39], as well as their conservation across species [40, 41], expression in different tissues and cell types, and their involvement in every biological process. miRNAs function as guide molecules by base pairing with the target mRNA, inducing translation repression or transcripts cleavage [42]. Consequently, miRNA deregulation is a hallmark of several pathological conditions, including cancer.
MetastamiRs are regulatory miRNAs which promote or suppress various steps in the migration and metastasis of cancer cells [24]. It seems that these metastasis-associated miRNAs do not influence primary tumors in either the development or initiation steps of tumorigenesis, but they regulate key steps in the metastatic program and processes, such as EMT, apoptosis, and angiogenesis. Emerging evidence continually recognizes tumor-specific metastamiRs in many tumor models, including breast, lung, prostate, colorectal, gastric, and head and neck cancer. However, in the context of neuroblastoma, no metastamiRs have been grouped or identified. Altered expression of select miRNAs (http://mircancer.ecu.edu) has been shown to exert a causal role in metastasis [20, 22, 23, 43-51]. It is noteworthy that these studies focus on a single miRNA manipulation approach, underscoring the metastatic response through a single gene target. Considering the complexity of sequential steps involved in metastasis, it is necessary to ascertain all causal metastamiRs that play crucial roles in NB metastasis. To our knowledge, this is the first of such an endeavor in the neuroblastoma setting. The results of this study comprehensively identified 74 metastamiRs from whole genome miRNA profiles of a manifold of metastatic tumors from a unique clinically translatable mouse model of aggressive high-risk NB. Further, this study determined the translation of these metastamiR reorganizations into the functional downstream response, that is, target proteins translation that defines metastasis, and the influential role of metastimiRs in clinical outcomes.

\section{Results}

\section{Spontaneous and reproducible high-risk metastatic disease in vivo}

Human neuroblastoma cell-line, SH-SY5Y is the third sub-line of SK-N-SH (SK-N-SH $\rightarrow$ SH-SY $\rightarrow$ SH-SY $5 \rightarrow$ $\mathrm{SH}-\mathrm{SY} 5 \mathrm{Y}$ ) that contains both neuroblast-like (N-type) floating and substrate-adherent (S-type) epithelial-like cells [52]. More importantly, SH-SY5Y cells are unique MYCN non-amplified cells. Xenotransplantation of SHSY5Y cells resulted in the development of $\sim 200 \mathrm{~mm}^{3}$ xenografts in $\sim 70 \%$ of the animals within 30 days (Fig. 1a) $[53,54]$. About $30 \%$ of the mice that received identical clones initially (within 10-20 days) showed xenograft development, then subsided to a residual tumor (Fig. 1a). However, over an extended 50-60 day period, these mice suddenly gained weight and presented with multiple clinically-mimicking metastatic tumors in the mediastinum and retroperitoneal, pelvic, abdominal, and chest cavities (Fig. 1a). These mice generally produced 5-12 large, viable, often multi-lobular tumors in multiple sites with well-organized blood supplies (Fig. 1a). This aggressive disease with metastatic dissemination developed over a short period (1-2 weeks) and vigorously, as 

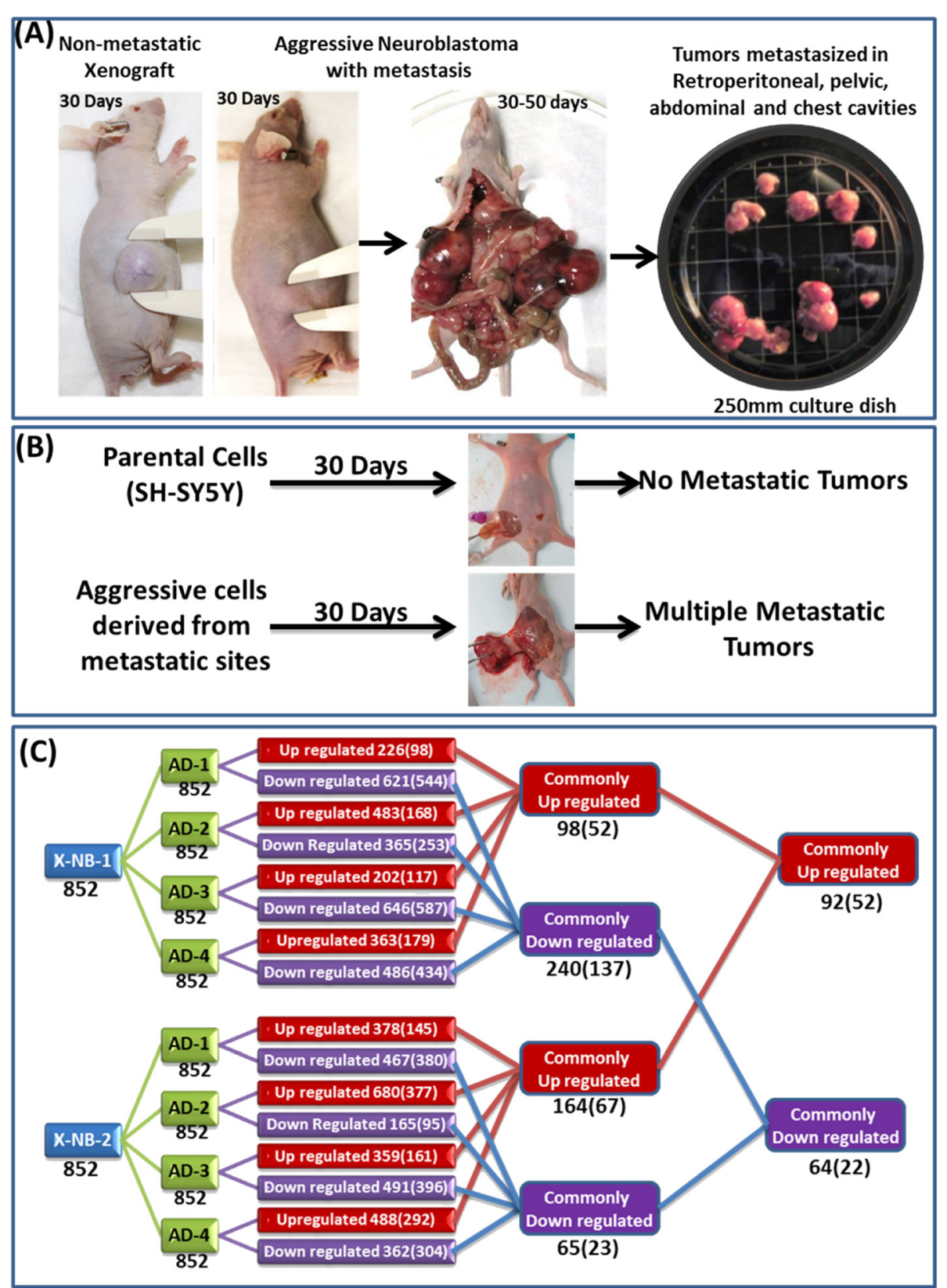

Fig. 1 Comparative miRNA profiling in non-metastatic primary xenografts and the manifold of metastatic tumors from animals bearing aggressive neuroblastoma. a Representative mice showing non-metastatic xenograft and high-risk aggressive metastatic disease. Plate showing tumors harvested from multiple metastatic sites. b Schematic representation showing relative tumorigenic capacity and aggressive disease formation of parental SH-SY5Y and metastatic site derived aggressive cells (MSDACs). Subcutaneously injecting MSDACs produced large xenograft with multiple metastatic tumors in retroperitoneal, pelvic, abdominal, and chest cavities. c Traverse analysis of whole genome miRNA expression between non-metastatic (X-NB) and aggressive disease (AD) animals. Total of 852 miRNAs were compared between groups and their alterations are color coded (Red - upregulated; Blue - down regulated). Total number of altered molecules under each comparison is provided in the corresponding box. Numbers in the parenthesis are the molecules that are significantly ( $>$ or $<2$ fold) modulated

evidenced by time-lapse non-invasive fluorescence imaging. Parallel xenotransplanted animals with no tumor dissemination to distant sites over the extended period served as the non-metastatic controls.

Metastatic Site Derived Aggressive cell (MSDAC) clones derived from the manifold of metastatic tumors were discretely characterized by karyotyping, whole genome array CGH analysis, and tumorosphere-forming capacity (data reported elsewhere). MSDACs are relatively small and spherical with thin neurites. Administration of MSDACs produced $>500 \mathrm{~mm}^{3}$ tumors (wet weight $2.40 \pm 1.30 \mathrm{~g}$ ), compared with the $<150 \mathrm{~mm}^{3}$ xenografts $(0.20 \pm 0.04 \mathrm{~g})$ 
resulting from parental cells within 30 days (Fig. 1b). The mice that received MSDACs presented with multiple metastatic tumors in the retroperitoneal, pelvic, abdominal, and chest cavities, demonstrating the reproducibility of the aggressive disease. Conversely, the mice that received parental cells did not exhibit any distant metastasis.

\section{Reorganization of metastamiRs in high-risk metastatic disease}

To define the miRNAs' modulations in the evolution of high-risk neuroblastoma and to identify crucial players (metastamiRs) that orchestrate metastasis, we adopted a whole genome miRNA profiling approach. A total of 852 functional transcripts were assayed in triplicate for each condition. Traverse analysis between the non-metastatic controls to the manifold of metastatic tumors not only recognized unique animal/tumor-specific expression signatures, but also identified clusters of miRNAs that were commonly up-or downregulated (Fig. 1c). Overall, of the 852 transcripts analyzed, we observed an upregulation of 226, 483, 202, 363 (when compared with X-NB-1), 378, 680, 359, and 488 (compared with X-NB-2) miRNAs in metastatic tumors. Applying stringent criteria ( $>2$ fold), a total of 98, 168, 117, 179 ( $\nu$ s. X-NB-1), 145, 377, 161, and 292 ( $v s$. X-NB-2) miRNAs were significantly upregulated in the metastatic tumors. Evidently, 52 (vs. X-NB1 ) and 67 ( $v$ s. X-NB-2) miRNAs were upregulated across all the metastatic tumors investigated. Upregulation of 52 miRNAs remained consistent across the metastatic tumors, irrespective of the comparisons to the nonmetastatic controls (Fig. 2). Conversely, a total of 621, 365, 646, 486 (vs. X-NB-1), 467, 165, 491, and 362 (vs. $\mathrm{X}-\mathrm{NB}-2)$ miRNAs were downregulated in metastatic tumors. Significantly ( $>2$ fold), 544, 253, 587, 434 (vs. XNB-1), 380, 95, 396, and 304 (vs. X-NB-2) miRNAs were completely suppressed in the metastatic tumors. More importantly, 137 ( $v s$. X-NB-1) and 23 (vs. X-NB-2) miRNAs were downregulated across all metastatic tumors. Remarkably, downregulation of 22 miRNAs persisted across the metastatic tumors, irrespective of the traverse comparisons to the controls (Fig. 3a).

To validate the altered expression levels observed with whole genome miRNA array, we examined the expression levels of select miRNAs including hsa-miR-1260, hsamiR-1224-3p (showing significant upregulation; see Fig. 2), hsa-miR-93, hsa-miR-20a, hsa-miR-125b, hsa-miR-27b (showing significant downregulation; see Fig. 3a), using individual miRNA QPCR analysis. Compared to the nonmetastatic xenograft, we observed a complete $(P<0.001)$ decrease in the expression of hsa-miR-93, hsa-miR-20a, hsa-miR-125b, and hsa-miR-27b (Fig. 3b). Conversely, we observed a significant $(P<0.001)$ upregulation of hsamiR-1260 and hsa-miR-1224-3p in metastatic tumor compared with the non-metastatic control (Fig. 3b). These data are consistent with the observed expression levels of these miRNAs using the whole genome approach.

\section{Altered expression of the target (metastasis-related) proteins validates the translation of the functional response of the neuroblastoma metastamiRs}

To further substantiate our findings, we investigated the expression levels of target proteins of the identified metastamiRs. Since metastasis is a complex processes, and as we identified a total of 74 metastamiRs in this setting, we examined a total of 49 targets that are involved in tumor progression, i.e., metastasis, in MSDACs and a manifold of metastatic tumors. MetastamiRs regulating these targets and their expression status in our study are presented in Table 1 (color-coded). In MSDACs grown ex vivo, we observed an induced expression of ADAMTS-1, CASP8, CDK4, CTGF, CYCLIN-D1, ELK1, ESR1, CFOS, FRA, IL-1 $\alpha$, JUND, kRAS, MCP1, MMP2, MMP3, MMP9, MMP10, cMYC, SELE, TNF $\alpha$, and VEGF, and phosphorylation of AKT1/ 2/3, NOS3, p38, and EGFR compared with the parental SH-SY5Y cells (Fig. 4a). Compared with the nonmetastatic xenograft controls, we observed a robust increase in ADAMTS-1, ASK1, AURK $\beta$, Birc1, Birc2, Birc5, CD54, CDK4, CTGF, CXCR4, CYCLIN-D1, EGFR, ELK1, ESR1, CFOS, FRA, GRB10, pGSK3 $\beta$, IL1 $\alpha$, JUND, KRTAP1-1, MEGF10, MMP2, MMP3, MMP9, MMP10, MTA2, MYB, cMYC, NF2, P21, PTPN3, CLEAVED PARP, PKC, SDF-1 $\beta$, SEMA3D, SELE, STAT3, TNF $\alpha$, TNFR1, and VEGF expression, as well as AKT1/2/3 and NOS3 phosphorylation in the manifold of metastatic tumors (Fig. $4 \mathrm{~b}$ and c).

We then sought to define and typify the target alterations in aggressive neuroblastoma. We used a custommade TMA constructed with a manifold of tumors from the metastatic sites of several animals coupled with nonmetastatic xenograft controls. These were subjected to automated IHC and image analysis for select targets, including MEGF10, KRTAP1-1, SEMA3D, MYC, and GRB10. Consistent with our immunoblotting observations under ex vivo and in vivo conditions, MEGF10, KRTAP1-1, SEMA3D, MYC, and GRB10 IHC staining revealed relatively strong positivity in metastatic tumors (Fig. 5). Multiple EGF-like-domains-10 (MEGF10) positive staining appeared in brown, and was predominantly localized in plasma membranes in a punctuated pattern (Fig. 5). MEGF10 immunoreactivity was barely detectable in non-metastatic NB xenografts. However, the manifold of metastatic tumors exhibited significantly high immunoreactivity for MEGF10 localization (Fig. 5). Keratin-associated protein family 1-1 (KRTAP1-1) positivity appeared in brown, and was predominantly localized in the cytoplasm (Fig. 5). Consistent with our immunoblotting data, we observed a profound and 

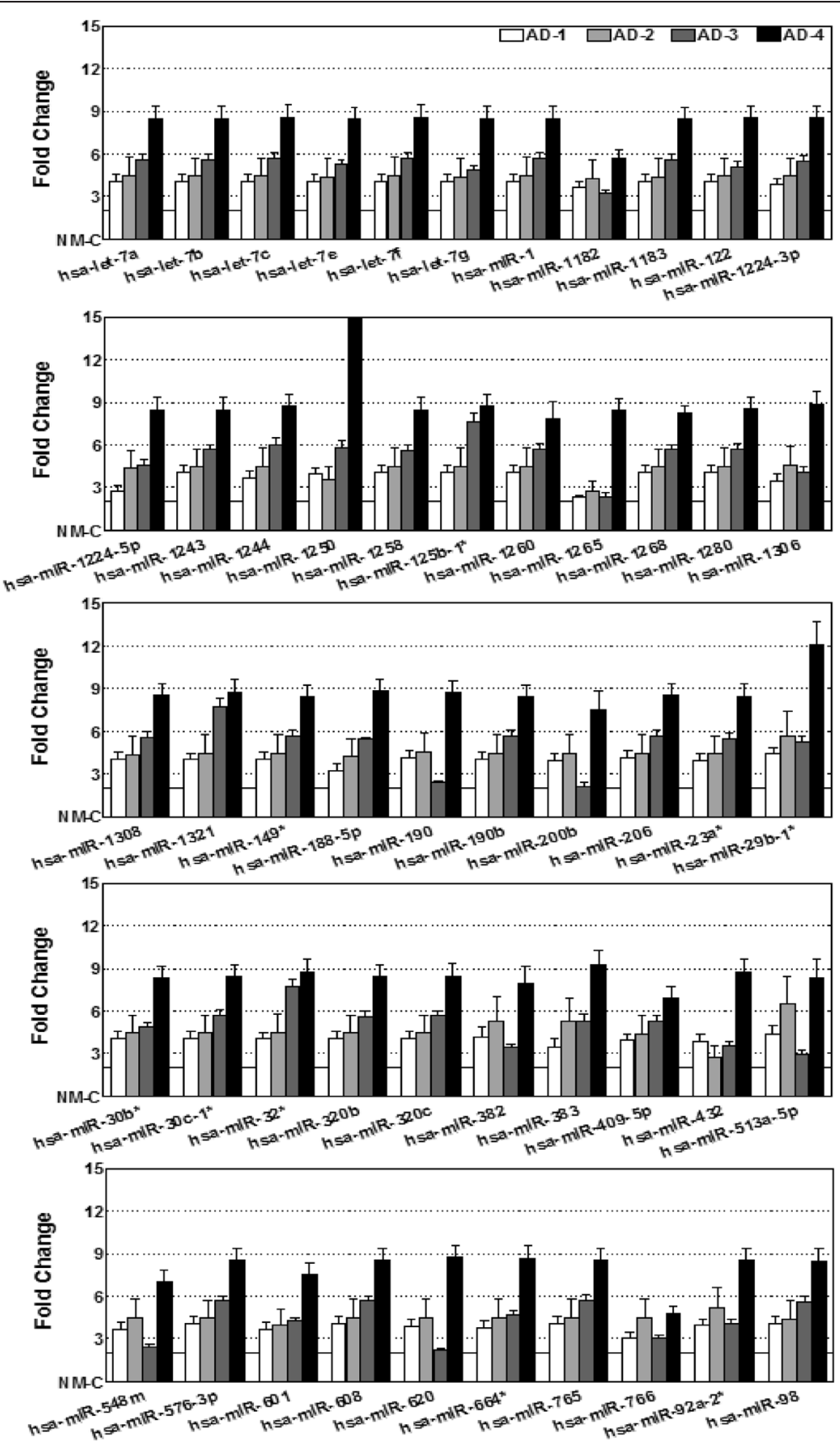

Fig. 2 Activated miRNAs in high-risk metastatic neuroblastoma. Histograms showing the expression profile of 52 miRNAs that were significantly $(>2$ fold) up regulated across the metastatic tumors of the animals with aggressive disease. Data mining was performed using traverse analysis comparing each non-metastatic control to that of the aggressive disease profiles

significant $(P<0.001)$ increase in the expression of KRTAP1-1 in the metastatic tumors as opposed to the non-metastatic controls (Fig. 5). Likewise, Semaphorin3D (SEMA-3D) IHC exhibited moderate cytoplasmic positivity with a dark brown color (Fig. 5). Compared with the non-metastatic controls, increased expression of SEMA-3D was evident in almost all metastatic tumors analyzed. MYC-IHC revealed strong nuclear positivity in all tumors analyzed. We observed a marginal increase in MYC expression in the metastatic tumors when compared with non-metastatic controls (Fig. 5). Growth factor receptor-bound protein 10 (GRB10) IHC revealed high levels of localization in nonmetastatic neuroblastoma tissues. Notably, immunoreactivity was highly intense and significant $(P<0.001)$ in metastatic tumors (Fig. 5). The positive staining of 

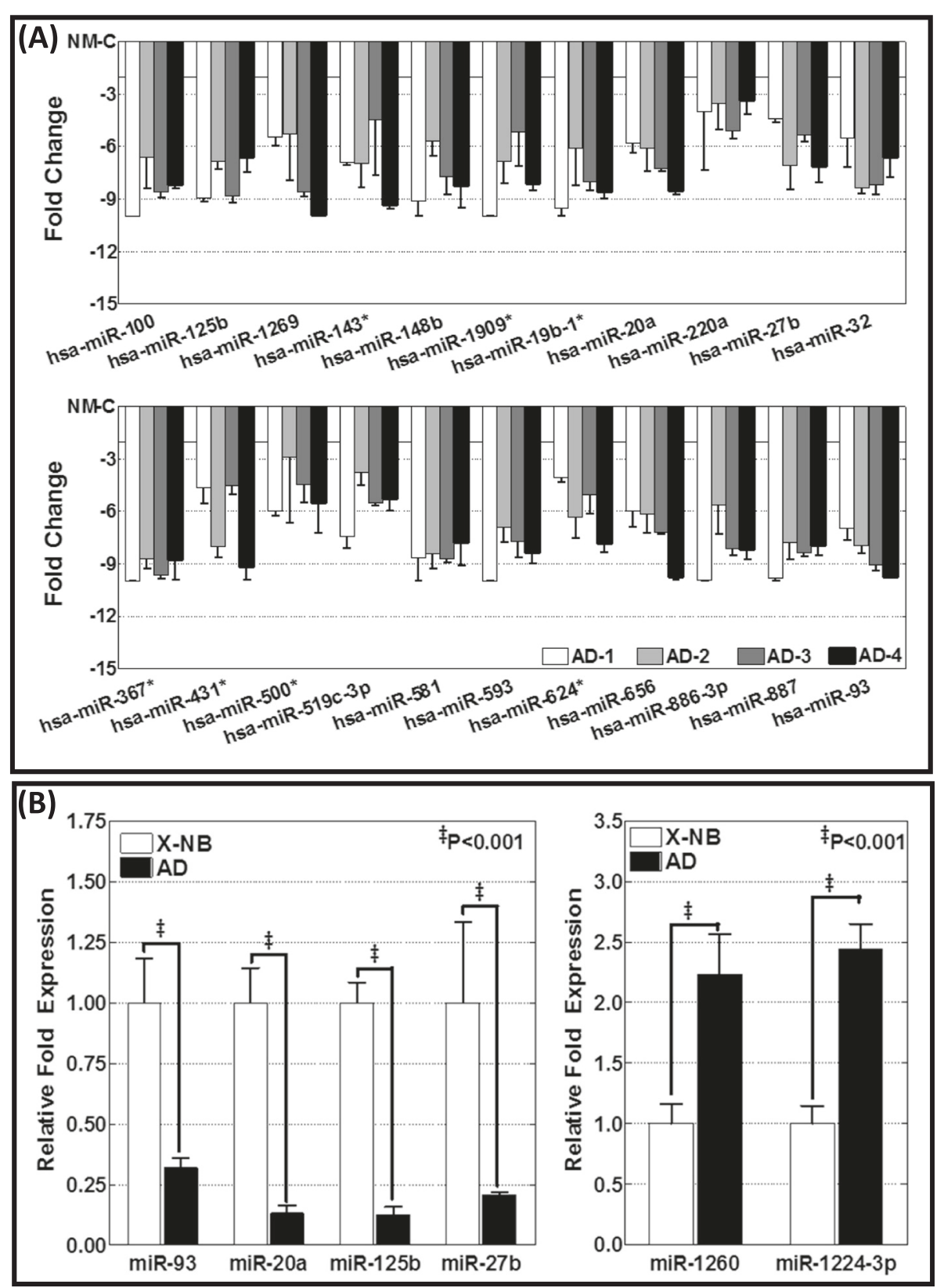

Fig. 3 Regulated miRNAs in high-risk metastatic neuroblastoma. a Histograms showing the expression profile of 22 miRNAs that were significantly ( $<2$ fold) down regulated across the metastatic tumors of the animals with aggressive disease. Data mining was performed using traverse analysis comparing each non-metastatic control to that of the aggressive disease profiles. $\mathbf{b}$ miRNA RT-qPCR analysis showing the complete regulation of Hsa-miR-93, Hsa-miR-20a, Hsa-miR-125b and Hsa-miR-27b and, significant increase in the expression of Hsa-miR-1260 and Hsa-miR-1224-3p in metastatic tumors as compared to the non-metastatic xenograft

GRB10 appeared in dark brown, and was predominantly localized in the cytoplasm.

These observations were consistent across tumors from the same animal as well as tumors from different animals. We observed similar alterations in the expression of MEGF10, KRTAP1-1, SEMA3D, MYC, and GRB10 in the manifold of distant tumors reproduced from aggressive disease-bearing animals. Aperio image analysis coupled with PRISM stats were used to demonstrate the increased expression of these target proteins in the tumors of animals with aggressive disease, but not in the non-metastatic xenografts (Fig. 5). Ex vivo and in vivo upregulation of these targets, which are well-documented candidates for metastasis and tumor 
Table 1 Targets analyzed by the western-blot and the expression status (color coded) of the corresponding metastamiRs in highrisk metastatic neuroblastoma

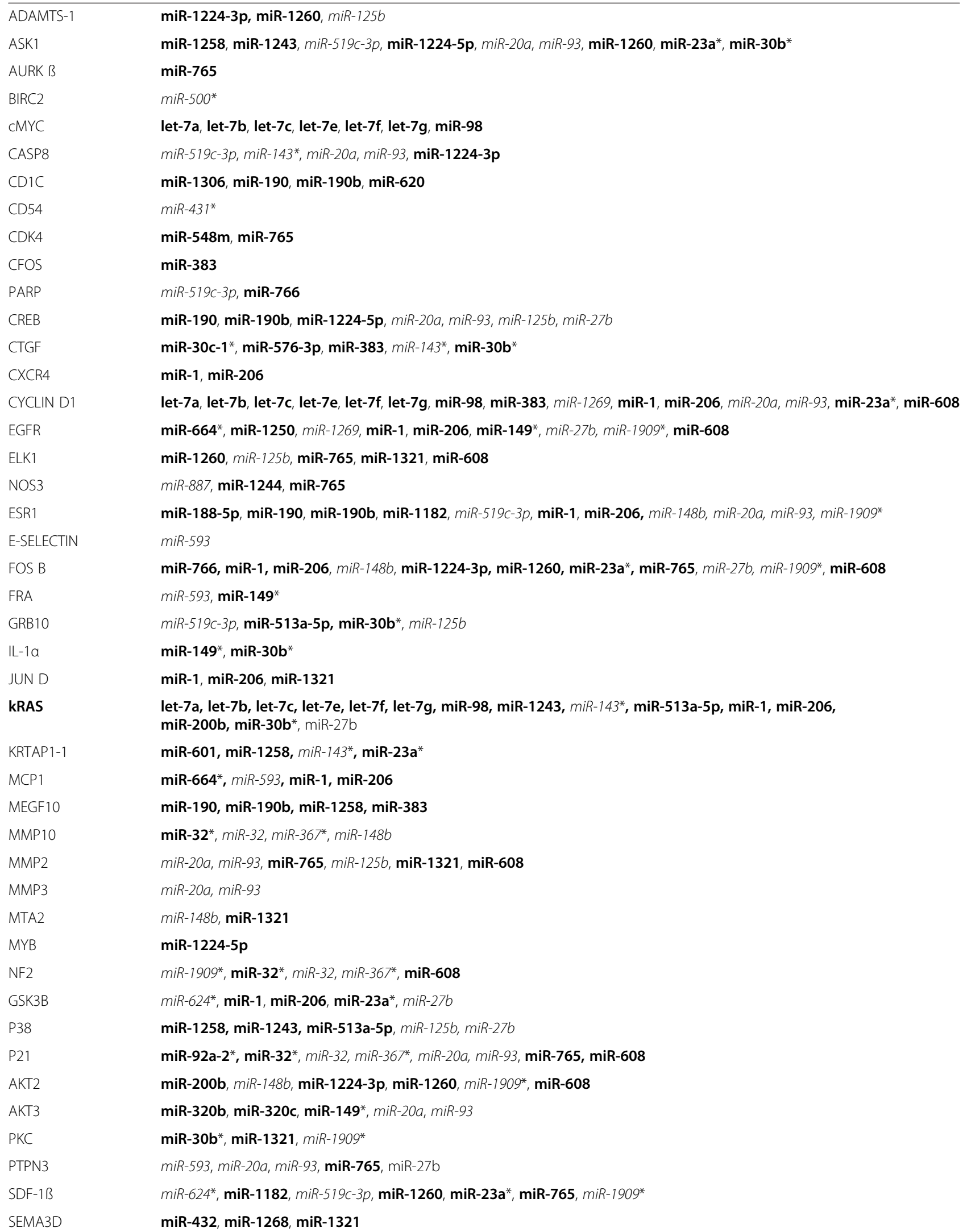


Table 1 Targets analyzed by the western-blot and the expression status (color coded) of the corresponding metastamiRs in highrisk metastatic neuroblastoma (Continued)

\begin{tabular}{ll}
\hline STAT3 & let-7a, let-7b, let-7c, let-7e, let-7f, let-7g, miR-98, miR-1244, miR-20a, miR-93, miR-125b, miR-1909*, miR-608 \\
SURVIVIN & miR-1182, miR-1321 \\
TNFR1 & miR-766 \\
TNF-a & miR-125b-1*, miR-519c-3p, miR-1909* \\
VEGF & miR-383, miR-1, miR-206, miR-200b, miR-20a, miR-125b, miR-27b \\
\hline
\end{tabular}

Up regulated; Down Regulated
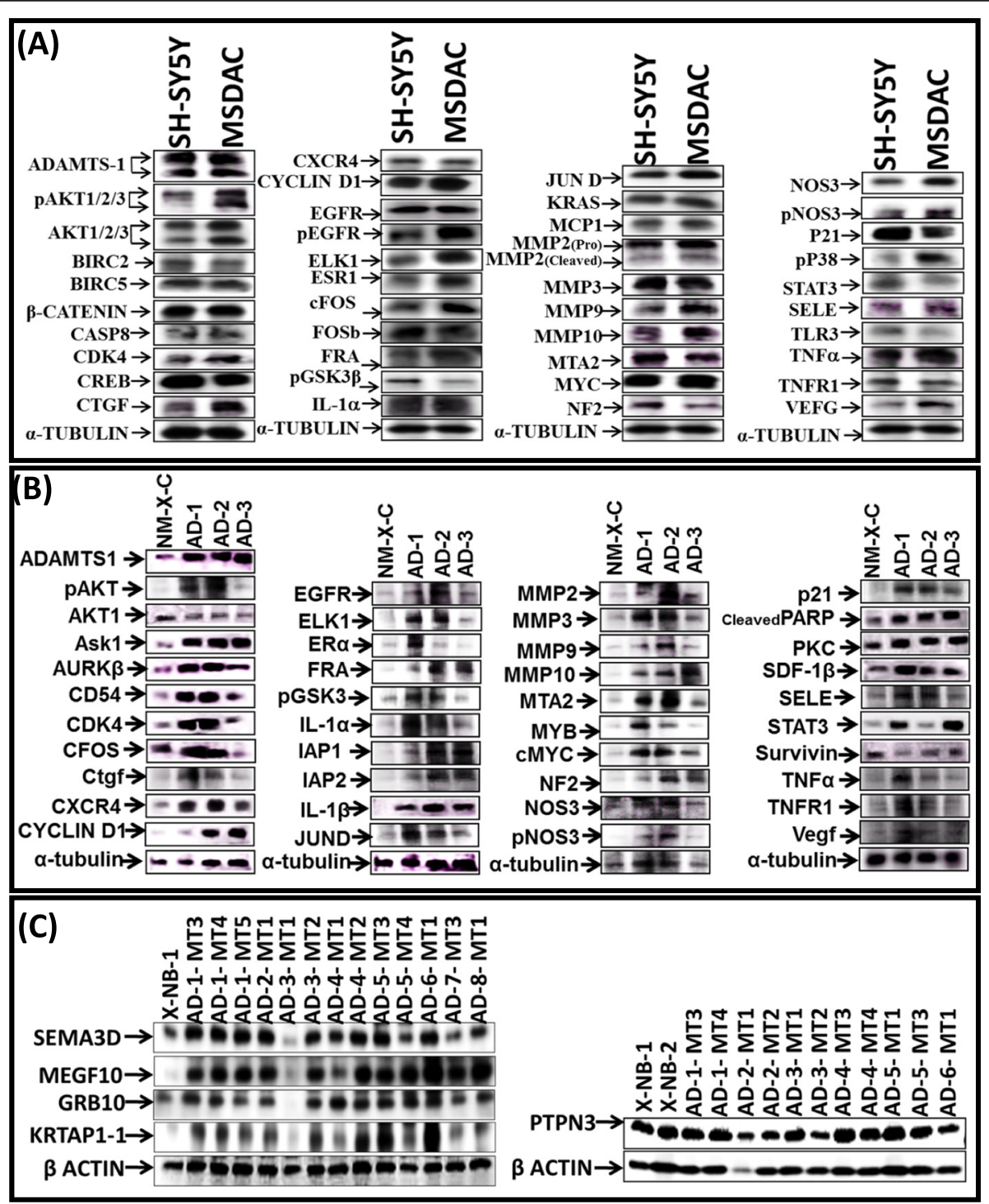

Fig. 4 Modulation of metastamiRs target proteins in MSDACs ex vivo and in metastatic tumors in vivo. Immunoblots showing the expressional modulation of key metastatsis and tumor progression related protein targets of the identified metastamiRs in (a) parental SH-SY5Y cells vS MSDACS under ex vivo conditions and; (b and $\mathbf{c}$ ) non-metastatic xenograft vs manifold of metastatic tumors from aggressive disease animals in vivo 


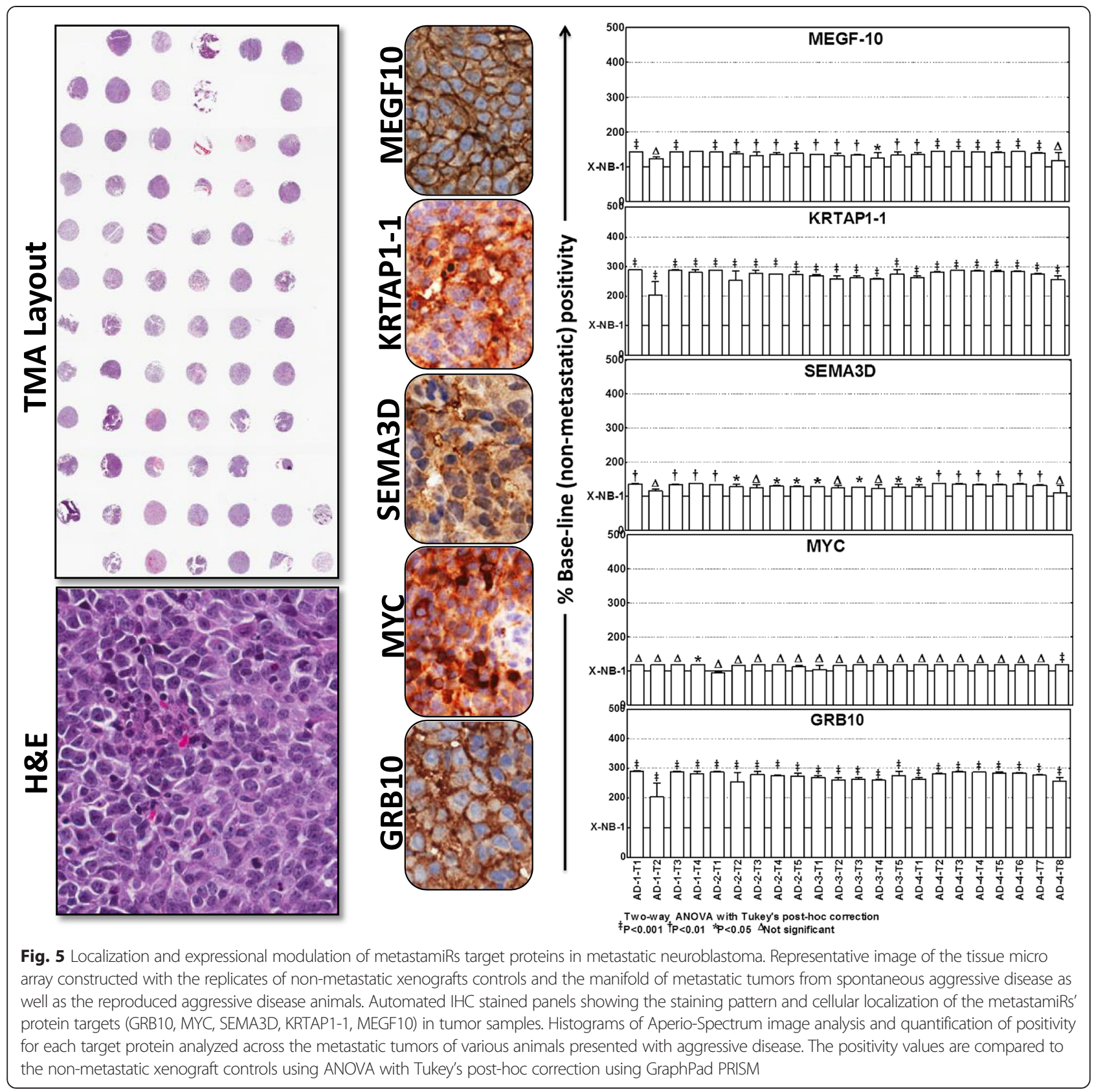

progression of the metastamiRs that were identified in this study, underscores the translation of the functional response (metastasis) of metastamiRs in this setting.

\section{Altered miRNAs dependent regulation of functional targets}

Further to define the direct role of the deregulated miRNAs of high-risk aggressive disease in the regulation of the target proteins that demonstrated profound alterations both in the metastatic tumors in vivo and in MSDACs ex vivo we examined the corresponding alterations of the target proteins after functionally mimicking or inhibiting miRNAs. First, MSDACs transiently transfected with mimics for hsa-miR-125b, hsamiR-27b, hsa-miR-93 or hsa-miR-20a (those exhibited complete suppression in aggressive disease) and examined for the regulation of their corresponding target proteins (Fig. 6a). High-throughput quantitative confocal immunofluorescence demonstrated a significant $(\mathrm{P}<0.001)$ inhibition of MMP2, p38, TNFa and VEGF in MSDACs in the presence of hsa-miR-125b mimic (Fig. 6bi). In addition, we observed a marginal decrease in GRB10 and STAT3 expression with hsa-miR-125b mimic. Similarly, functionally mimicking hsa-miR-27b 


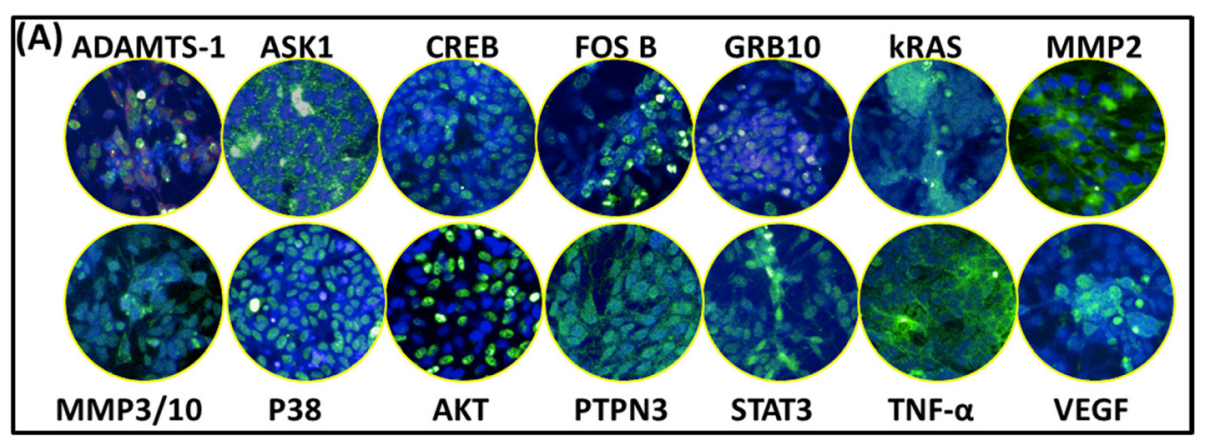

(B) $\square$ Control $\square$ Mimic ${ }^{\ddagger} P<0.001 \dagger^{\dagger}<0.01 \Delta_{\text {Not Significant }}$
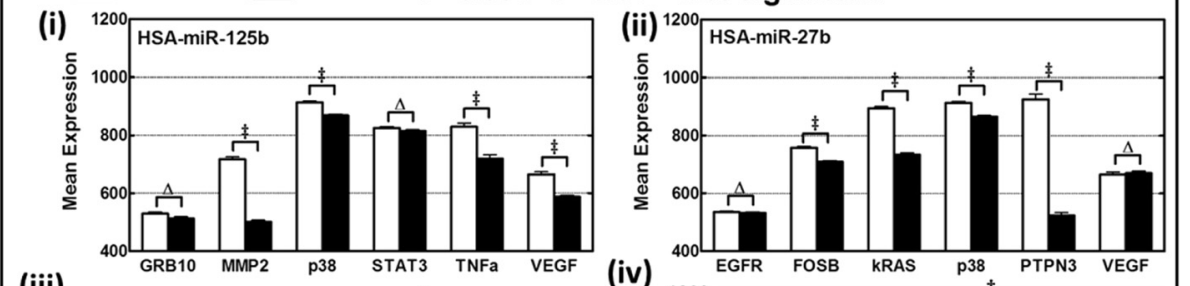

(iii)
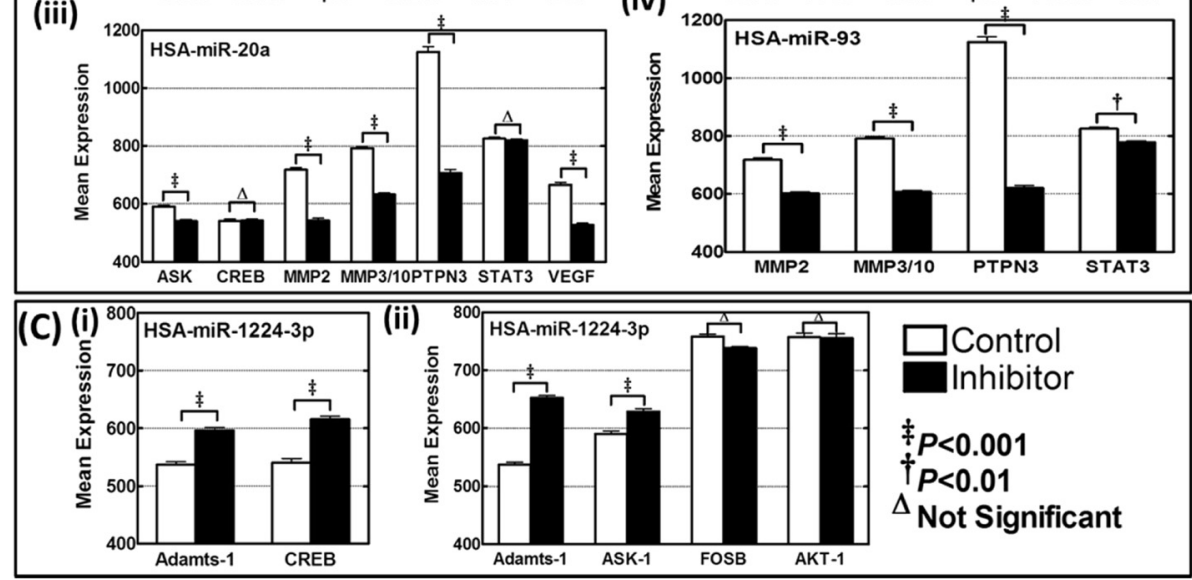

Fig. 6 MetastamiRs dependent regulation of functional protein targets. a Operetta high-content confocal imaging showing immunofluorescence of ADAMTS-1, ASK-1, CREB, FOSB, GRB10, kRAS, MMP2, MMP3/10, p38, AKT, PTPN3, STAT3, TNFa and VEGFa in MSDACs. b Histograms of mean cell-Alexa Fluor intensity obtained from Columbus automated batch analysis showing alterations in the expression (i) GRB10, MMP2, p38, STAT3, TNFa and VEGF in cells with hsa-miR-125b mimic, (ii) EGFR FOSB, kRAS, p38, PTPN3 and VEGF in hsa-miR-27b mimic transfected cells, (iii) ASK1, CREB, MMP2, MMP3/10, PTPN3, STAT3and VEGF in MSDACs with hsa-miR-20a mimic and, (iv) MMP2, MMP3/10, PTPN3 and STAT3 with hsa-miR-93 mimic in MSDACs. c Histograms of mean cell-Alexa Fluor intensity showing alterations in the expression of (i) ADAMTS-1 and CREB with hsa-miR-1224-3p inhibition and, (ii) ADAMTS-1, ASK1, FOSB and AKT-1 with hsa-miR-1260 inhibition. Group-wise comparisons were performed with ANOVA with Tukey's post-hoc correction

resulted in the profound $(\mathrm{P}<0.001)$ inhibition of FOSB, kRAS, p38 and PTPN3 (Fig. 6bii). Interestingly, mimicking hsa-miR-27b did not inhibit the expression of EGFR and VEGF in MSDACs. On the other hand, MSDACs transfected with hsa-miR-20a exhibited significant $(\mathrm{P}<$ 0.001) inhibition of ASK1, MMP2, MMP3/10, PTPN3 and VEGF (Fig. 6biii). We did not see any consistent inhibition of CREB and STAT3 at least with the mimic for hsa-miR-20a. Moreover, hsa-miR-93 mimic exhibited statistically significant inhibition of MMP2, MMP3/10, PTPN3 and STAT3 in this setting (Fig. 6biv). Next, MSDACs transiently transfected with inhibitors for hsamiR-1224-3p or hsa-miR-1260 (both showed profound induction in metastatic tumors) and examined for the alterations in protein targets. Inhibiting hsa-miR-1224$3 \mathrm{p}$ resulted in the significant $(\mathrm{P}<0.001)$ induction of ADAMTS-1 and CREB (Fig. 6ci). Like-wise, inhibiting hsa-miR-1260 markedly $(\mathrm{P}<0.001)$ induced ADAMTS-1 and ASK1 (Fig. 6cii). However, inhibiting hsa-miR-1260 did not result in the induction of FOSB and AKT-1 in this setting. Together, these results clearly demonstrate the direct role of altered miRNAs observed in high-risk aggressive disease in the regulation of the protein targets identified in this setting and, thereby validates the translation of functional response of the neuroblastoma metastamiRs. 


\section{Altered miRNAs serve as metastamiRs}

To determine the relevance of metastamiRs in aggressive disease, we first clarified their role in cancer biological functions, network and communal molecular orchestrations, and tumor progression. For the miRNAs that were reorganized in high-risk NB, IPA "Core-Analysis" identified multiple networks classifying their roles in diseases including cancer and their specific functions (Additional file 1: Figure S1). Functionally, these miRNas were shown to be intrinsically involved in cell death and cell survival, inflammation, the cell cycle, cellular movement, DNA replication, recombination and repair, cell-to-cell signaling and interaction, and cellular growth and proliferation. These processes play defined roles in metastasis (Additional file 1: Figure S1). Convergence of all of the miRNA networks at the processes that endorse metastasis identify the altered miRNAs as metastamiRs. To further validate whether these miRNAs are recognized as metastamiRs in other tumor systems, we used the cancer miRNA profile database from mircancer.ecu.edu (release version of miRCancer June 2014), ingenuity pathway analysis of diseases and biological functions, and a manual literature survey.

Of the 74 metastamiRs, data mining in miRCancer identified at least 36 that were altered: hsa-let-7a, hsa-let-7b, hsa-let-7c, hsa-let-7e, hsa-let-7f, hsa-let-7 g, hsa-mir-1, hsa-mir-100, hsa-mir-122, hsa-mir-1250, hsa-mir-1258, hsa-mir-125b, hsa-mir-1280, hsa-mir-143, hsa-mir-148b, hsa-mir-149, hsa-mir-190b, hsa-mir-200b, hsa-mir-206, hsa-mir-20a, hsa-mir-220a, hsa-mir-23a, hsa-mir-27b, hsa-mir-29b-1, hsa-mir-30b, hsa-mir-30c-1, hsa-mir-32, hsa-mir-383, hsa-mir-432, hsa-mir-500, hsa-mir-601, hsamir-656, hsa-mir-765, hsa-mir-886-3p, hsa-mir-92a, and hsa-mir-93. Alterations in these metastamiRs were found in one or more of the following tumor systems: acute lymphoblastic leukemia, acute myeloid leukemia, acute promyelocytic leukemia, b-cell lymphoma, bladder cancer, breast cancer, bronchioloalveolar carcinoma, cervical cancer, cholangiocarcinoma, chronic lymphocytic leukemia, colon cancer, endometrial cancer, esophageal squamous cell carcinoma, follicular cancer, gastric cancer, glioblastoma, head and neck squamous cell carcinoma, hepatocellular carcinoma, laryngeal squamous cell carcinoma, liver cancer, lung cancer, malignant melanoma, mantle cell lymphoma, medulloblastoma, mesenchymal cancer, nasopharyngeal cancer, neuroblastoma, non-small cell lung cancer, oral squamous cell carcinoma, osteosarcoma, ovarian cancer, ovarian carcinoma, pancreatic cancer, papillary thyroid carcinoma, pituitary carcinoma, prostate cancer, renal cell carcinoma, thyroid cancer, and tongue cancer (data not shown).

Ingenuity pathway analysis, however, identified the expressional association of these metastamiRs in at least eighty different tumor systems (Additional file 2: Figure S2).
Armed with the above information, we further explored the crucial role of metastamiRs in tumor cell metastasis. Additional file 5: Table S1 shows the list of identified miRNAs, their functions in the context of metastasis and tumor progression as per the published evidence, total number of their gene targets, and the references from which we obtained this information. Interestingly, almost all of the miRNAs identified in our high-risk metastatic neuroblastoma have been well characterized in terms of metastasis regulation (Additional file 5: Table S1), which could identify them as metastamiRs.

\section{Alterations in the targets of the identified metastamiRs are associated with poor clinical outcomes}

To demonstrate the functional relevance of these metastamiRs to high-risk metastatic neuroblastoma and poor clinical outcomes, we examined the correlation of individual expression of their gene targets with overall survival (OS) in patients with neuroblastoma. Utilizing the gene expression data for a cohort of 88 human neuroblastoma patients, we examined the prognostic values for a total of 22 gene targets that had validated expression levels in our model. Kaplan-Meier plots showed a significant association between increased expression of ELK1, CDK4, MMP2, AURK $\beta$, FRA, MYB, JUND, BIRC5, AKT2, SELE, TNFa (Additional file 3: Figure S3), NOS3, ESR1, KRTAP1-1, MMP3, MMP9, NF2, CXCR4, ADAMTS1, VEGF, CD54, EGFR, and AKT1 (Additional file 4: Figure 4) and poor OS in patients with neuroblastoma. This inverse association also reflects poor relapse-free survival in these patients (data not shown). Interestingly, there was a definite association between CREB1 loss and poor OS (Additional file 3: Figure 3). This CREB1 loss also resulted in poor relapse-free survival in children with neuroblastoma. Clinical outcome association analysis revealed a strong correlation between the increased expression of the genes listed above and stage progression, favorable $\rightarrow$ unfavorable disease and alive $\rightarrow$ died-of-disease (data not shown). Together, these data demonstrate the alterations of gene targets by the reorganization of metastamiRs in high-risk metastatic disease that drives poor clinical outcomes in children with neuroblastoma.

\section{Discussion}

About $60 \%$ of neuroblastoma patients with high-risk disease will relapse with hematogenous metastasis [6], despite intensive multimodal therapy [3, 5, 7-14]. Neuroblastoma is derived from embryonic neural crest cells that have a high potential to migrate. Since metastatic NB has a high mortality rate, understanding the molecular mechanism flow-through that is involved in neuroblastoma cell invasion and metastasis will help us 
to design more effective therapies against metastatic NB, particularly when we use appropriate clinically translatable animal models. In vitro and in vivo approaches have identified numerous molecular markers that play crucial roles in the functional orchestration of neuroblastoma metastasis [19, 55-58]. After $\mathrm{Ma}$ and colleagues [32] demonstrated that miRNAs were the upstream regulators of the complex invasion-metastasis network in breast cancer, researchers began to uncover the roles of miRNA(s) in highly metastatic neuroblastoma $[59,60]$. Transcriptome regulation occurs via miRNAs through multiple processes, including translational inhibition, destabilization, or RNA decay [33], all of which are considered important modulators of signal transduction pathways in metastatic progression.

Studies delineating the regulatory role of the miRNA in neuroblastoma metastasis have thus far been limited to the understanding of a single miRNA-associated target molecule's dependent functions along the axis of metastasis $[22,43,45,46,61]$. Considering the complexity of the sequential steps involved in neuroblastoma metastasis, it is crucial to define the functional role of every miRNA (metastamiR) that plays a vital role in each step of metastasis. For the first time, utilizing a clinically translatable animal model of high-risk metastatic neuroblastoma, we have identified the cluster of metastamiRs, their functional translation in terms of target protein expression, and the association of such alterations with clinical outcomes.

In the current study, we used multiple complementary methodologies to identify neuroblastoma metastamiRs. First, this study employed a unique clinically translatable, reproducible mouse model of human high-risk metastatic neuroblastoma coupled with whole genome miRNA array technology. We screened differentially expressed miRNAs in metastatic tumors as opposed to non-metastatic tumors. We observed significant interanimal variations in up-and downregulated miRNAs and under less stringent and more stringent conditions (see Fig. 1c). Examining this topic further, we validated the most distinctly deregulated over- and under-expressed miRNAs by RT-qPCR, utilized traverse analysis of altered miRNAs between the sample groups, characterized the reorganized miRNAs by compiling their interactions in ingenuity pathway analysis, expression in multiple tumor systems, and their functional role in metastasis and tumor progression. Further, we performed miRNAtarget analysis, comprehensively identified the alterations in the expression and cellular localization of the target proteins in ex vivo and in vivo metastatic cells, recognized the direct influence of the altered miRNAs on the regulation of target proteins with mimic/inhibitor approach, and compiled the target proteins' association with neuroblastoma patient outcomes.
Since the main objective was to identify the defined metastamiRs of neuroblastoma, we compared the traverse examination of four aggressive disease expression profiles to that of non-metastatic profiles to derive clear evidence of differential expression without equivocal outcomes. Interestingly, the outcome of the present work confirmed some findings from other neuroblastoma miRNA studies (http://mircancer.ecu.edu/browse.jsp) [59], but also essentially identified many new metastamiRs in this setting. Of the 74 metastamiRs identified in this study, we found an overlap of 16 metastamiRs, including Hsa-miR-148b, Hsa-miR-23a, Hsa-miR-100, HsamiR-93, Hsa-miR-125b, Hsa-miR-98, Hsa-miR-92a, Hsa-miR-29b, Hsa-miR-30c, Hsa-let-7a, Hsa-let-7b, Hsa-let-7c, Hsa-let-7e, Hsa-let-7f, and Hsa-let-7g, with the findings of other researchers. To our knowledge, this is the first such attempt to identify all-inclusive miRNAs that could play defined roles in metastasis, their functional translation, and their association with clinical outcomes, at least in the neuroblastoma setting. Almost all studies that investigated miRNA roles in neuroblastoma metastasis focused on understanding a single molecule. As discussed above, our approach to identify new metastamiRs in the neuroblastoma setting is directly related to the lack of any comprehensive approach studies for neuroblastoma or other tumor types. Limited overlap with similar studies in other cancers [59] may reflect the use of different detection platforms, miRBase releases, or tumor models or origins. In addition, results that are based solely on microarray data have a relatively high false-positive rate. Thus, we validated our microarray results with RT-qPCR for upregulation (Hsa-miR-1260; Hsa-miR-1224-3p) and downregulation (Hsa-miR-20a, Hsa-miR-27b, Hsa-miR125b, Hsa-miR-93) profiles (see Fig. 3b).

Since it is important to characterize each of the reorganized miRNAs in neuroblastoma metastasis, and this activity was within the scope of this study, we extensively investigated the role of each miRNA in tumor progression and metastasis in multiple tumor systems, including neuroblastoma. Regulation of these miRNAs has been documented in many tumor systems and biological functions, including metastasis (Additional file 2: Figure S2). Though it is not practical to discuss each miRNA, we compiled their biological functions in the light of the published evidence that identifies them as metastamiRs (Additional file 5: Table S1). However, to understand their precise function in neuroblastoma metastasis and since expression profiles do not give us information about their biological functions, we measured the expressional alterations of key targets that were shown to play crucial roles in metastasis. For the first time, we quantified the expression and phosphorylation of 49 target proteins. Further, to capture the metastamiRs associated with translational 
alterations of the targets, we characterized their expression in metastatic cells ex vivo and in the manifold of aggressive metastatic tumors. The targets examined in the study are regulated by more than one metastamiR identified in this setting (see Table 1). Thus, to the best of our knowledge, the presented list of 49 deregulated metastasis-related protein targets of metastamiRs provides the most extensive list of neuroblastoma metastamiRs' associated response. In this respect, the global approach in our study to assess the expression data in the manifold of metastatic tumors, all in the screening phase by microarrays, in the validation phase by RT-qPCR and response validation by target protein expressions and their miRNA dependent regulation, as mentioned above, proved to be advantageous. We also applied clinical outcome association analysis with the validated protein targets of the identified metastamiRs in the neuroblastoma setting. Induced expression (ELK1, CDK4, MMP2, AURK $\beta$, FRA, MYB, JUND, BIRC5, AKT2, SELE, TNF- $\alpha$, NOS3, ESR1, KRTAP1-1, MMP3, MMP9, NF2, CXCR4, ADAMTS1, VEGF, CD54, EGFR, and AKT1) and suppression (CREB1) of metastamiRs' target proteins corresponded strongly with poor patient outcomes (Additional files 3: Figure S3 and Additional file 4: Figure S4).

\section{Conclusions}

For the first time, this study of miRNAs profiles in nonmetastatic and metastatic neuroblastoma identified a comprehensive list of 74 deregulated metastasis-associated miRNAs, termed metastamiRs. We primarily recognized new miRNAs associated with neuroblastoma metastasis, and also confirmed the results of other studies, as only a few miRNAs have been described as neuroblastoma metastamiRs. Further, our results demonstrated a significant modulation in the expression of 49 key metastasis-regulating protein targets of these metastamiRs in aggressive cells ex vivo and in the manifold of metastatic tumors in vivo. More importantly, the clinical outcome association analysis corresponded with poor overall and relapse-free survival. In conclusion, the current study identified a distinctive set of neuroblastoma metastamiRs that could serve as potential candidates for diagnostic markers for the switch from favorable to high-risk metastatic disease.

\section{Methods}

\section{Cell culture}

The SH-SY5Y human neuroblastoma cell line was obtained from ATCC (Manassas, VA) and was cultured and maintained as described earlier [54]. For passaging and for all experiments, the cells were detached using $0.25 \%$ trypsin $/ 1$ \% EDTA, re-suspended in complete medium, counted (Countess, Invitrogen), and incubated in a $95 \%$ air $/ 5 \% \mathrm{CO}_{2}$ humidified incubator.

\section{In vivo human neuroblastoma} experiments-development of reproducible nonmetastatic xenografts and mouse models of aggressive metastatic disease

All animal experiments conformed to American Physiological Society standards for animal care and were carried out in accordance with guidelines laid down by the National Research Council. All protocols were approved by the University of Oklahoma Health Sciences Center Institutional Animal Care and Use Committee and adhered to the ARRIVE guidelines. Seven-week-old athymic NCr-nu/nu nude mice (NCI, Frederick, MD) received SC injections of $5 \times 10^{6}$ SH-SY5Y cells suspended in Matrigel (BD Biosciences) into their right flank. Animals were observed for xenograft and/or aggressive metastatic disease development for extended periods of time (Min 15; Max 60 days). Tumor growth, regression, and dissemination to distant sites were investigated by tumor volume measurements and non-invasive fluorescent imaging. For this purpose, we administered 2 nmol/100 $\mu \mathrm{L}$ of IntegriSense 750 (Perkin Elmer, Inc., Waltham, Inc.) intravenously through the tail vein. After $24 \mathrm{~h}$, imaging was performed at excitation 745 and emission 800 using In Vivo Extreme (Carestream Health Inc., Rochester, NY). A reflectant image and x-ray CT image were also acquired to establish anatomical landmarks. Animals were euthanized by $\mathrm{CO}_{2}$ asphyxiation. The tumors from metastatic sites and non-metastatic xenografts were harvested and prepared as single-cell suspensions as described below. To reproduce high-risk aggressive disease, animals were injected with isolated and well-characterized clones of aggressive cells derived from individual metastatic sites, and observed for development of metastatic tumors. Parallel experiments were performed with cells derived and characterized from non-metastatic xenografts.

\section{Preparation of single-cell suspensions of tumor cells, cell sorting, and ex vivo maintenance}

To derive tumor cells from individual metastatic sites and non-metastatic xenografts, we thoroughly minced pre-weighed tumor samples in cell dissociation buffer (DMEM media supplemented with $10 \%$ FBS, penicillin/ streptomycin, $200 \mathrm{U} / \mathrm{mL}$ Collagenase type IV, and 0.6 $\mathrm{U} / \mathrm{mL}$ dispase). A $5 \mathrm{ml}$ serological pipet of titrated cell suspension was allowed to incubate at $37^{\circ} \mathrm{C}$ for $3 \mathrm{~h}$ for enzymatic dissociation, with mechanical dissociation every $15 \mathrm{~min}$. At the end of the incubation, cells were filtered through a $70 \mu \mathrm{m}$ filter and subjected to density centrifugation using Ficoll-Plaque Plus to separate viable cells from dead cells and tissue debris. Cells collected from the interface were diluted (1:3) in fresh DMEM:F12 with $10 \%$ FBS and counted for viable cells using Countess. Metastatic site derived aggressive cells (MSDACs) 
were grown ex vivo in stem cell medium (DMEM:F12 with $1 \%$ N2 Supplement, 2 \% B27 Supplement, $20 \mathrm{ng} / \mathrm{ml}$ hPDGF, $100 \mathrm{ng} / \mathrm{ml} \mathrm{EGF,} \mathrm{and} 1 \%$ antibiotic-antimycotic) at $37{ }^{\circ} \mathrm{C}, 5 \% \mathrm{CO}_{2}$. Suspensions were then sequentially characterized with karyotyping, whole genome array CGH analysis, ex vivo tumorosphere-forming capacity, and in vivo tumorigenicity.

\section{Total RNA/miRNA Isolation and whole genome miRNA microarray profiling}

Total RNA/miRNA from parental SH-SY5Y, MSDACs, and a manifold of metastatic tumors was extracted using TRIzol reagent (Invitrogen) or miRNeasy kit (Qiagen), following the manufacturer's protocol. RNA concentration and quality were determined using the RNA 6000 Nano assay on the Agilent 2100 Bioanalyzer (Agilent Technologies). miRNA expression profiles were performed using the human miRNA One Array V4 (Phalanx Biotech) that includes 1,884 unique miRNA probes and 144 experimental control probes. Each unique probe has 3 features. Probes contain $100 \%$ of Sanger miRBase V18 miRNA content. Isolated miRNA (500 ng) was labeled with Cy3 using a Kreatech ULS miRNA labeling kit and hybridized using an miRNA One Array V4 kit (Phalanx) following the manufacturer's protocol. Hybridized signals were detected by the microarray scanner G2600D (Agilent Technologies, Santa Clara, CA, USA) and quantified using ImageQuant (GE Healthcare Bio-Sciences, Pittsburgh, PA, USA). Complete matrix of the whole genome miRNA expression and their metadata are included in Additional files 6. Expression profiles of each feature were background-subtracted, normalized to internal U6 features, and compared between groups using Prism (GraphPad Software, Inc., La Jolla, CA, USA). To eliminate inter-animal variations that could equivocate expression outcomes within the group, we adopted non-metastatic control(s) $\rightarrow$ metastatic tumor(s) traverse analysis between every sample analyzed. For this, expression profile of background subtracted and array-normalized miRNAs of non-metastatic primary xenograft control-1 was first individually compared to the miRNA expression profiles of each aggressive metastatic tumors and, the overall and/or $>2$-fold up/down regulated miRNAs for each aggressive tumors are computed. The comparison analysis was then performed to identify miRNAs that were commonly up/ down regulated (both overall as well as $>2$-fold change) across the aggressive tumors as opposed to control-1. Next a similar detailed approach was utilized to obtain the miRNAs that were commonly up/down regulated (both overall and $>2$-fold) across the aggressive tumors as opposed to non-metastatic primary xenograft control2. Finally, crisscross data comparison analysis was performed between the data sets 1 and 2 to define the
miRNAs that were up or down regulated in aggressive metastatic tumors, ruling out any intra-group variations associated equivocal outcomes. Commonly reorganized metastamiRs (upregulated and downregulated) across the manifold of metastatic tumors are further examined Ingenuity Pathway analysis, Target Scan, and miRBase to determine the gene targets.

\section{MiRNA qPCR}

In order to validate the miRNA expression obtained from whole genome profiling, expression of selected metastamiRs, including hsa-miR-1224-3p, hsa-miR-1260 (both significantly upregulated), hsa-miR-125b, hsamiR-27b, hsa-miR-93 , and hsa-miR-20a (all significantly downregulated) were confirmed using QPCR. Hsa-miRU6 was used as an internal positive control. Briefly, poly (A) tailed (Poly (A) tailing kit, Life Technologies, Grand Island, NY, USA), miRNA was reverse transcribed using miRNA EasyScript ${ }^{\text {tw }}$ cDNA synthesis kit (Applied Biological Materials Inc., Richmond, BC, Canada) as per the manufacturer's protocol. QPCR sampling was performed in triplicate using an miRNA EasyScript ${ }^{\text {tw }}$ cDNA Synthesis Kit (Applied Biological Materials, Inc.) following the manufacturer's PCR conditions. U6 normalized expression was compared with the non-metastatic controls and expressed as a fold change. Group-wise comparisons were performed with two-way ANOVA with Tukey's post-hoc correction (GraphPad Prism). Similarly, correlations in the expression patterns obtained from the whole genome profiling with the QPCR results were performed using GraphPad Prism.

\section{Functional characterization of metastamiRs and target analysis}

To functionally characterize the identified miRNAs in tumor progression and metastasis we adopted three sequential approaches. First, utilizing Ingenuity Pathway Analysis (Ingenuity Systems, Inc.) we examined the credible intermolecular interactions and their association to cancer. Next, to underscore and identify metastamiRs' relevance in tumor progression and to delineate neuroblastoma-specific shifts, we used the miRCancer (http://mircancer.ecu.edu) database, and compared the expression profiles in various human cancers, including neuroblastoma. Lastly, by extracting information from the published literature, we examined the defined functions of the miRNAs in the context of metastasis and tumor progression. Three inter-linked databases, ingenuity pathway analysis, miRBase, and microRNA.org, were used for targeted analysis of the identified metastamiRs. Extracted lists of targets were subsequently compared to each other and select targets that played crucial roles in metastasis and were regulated by more than one 
metastamiR were identified and examined for translational modulations.

\section{Immunoblotting}

Total protein extraction and immunoblotting were performed as described earlier [62]. In MSDACs and metastatic tumors, we analyzed modulations in the altered expression of identified targets playing crucial roles in metastasis, including ADAMTS-1, pAKT1/2/3, AKT1/2/ 3 , ASK1, AURK $\beta$, Birc1, Birc2, Bric5, $\beta$-CATENIN, CASP8, CD54, CDK4, CREB, CTGF, CXCR4, CYCLIND1, EGFR, pEGFR, ELK1, ESR1, CFOS, FOSB, FRA, GRB10, pGSK3 $\beta$, IL1 $\alpha$, JUND, kRAS, KRTAP1, MCP1, MEGF10, MMP2, MMP3, MMP9, MMP10, MTA2, MYB, cMYC, NF2, NOS3, pNOS3, P21, pP38, PTPN3, CLEAVED PARP, PKC, SDF-1 $\beta$, SEMA3D, SELE, STAT3, TLR3, TNF $\alpha$, TNFR1, and VEGF. Blots were stripped and reblotted with either anti- $\alpha$-tubulin or anti$\beta$-actin to determine equal loading of samples.

\section{Tissue microarray construction and quantitative immunohistochemistry}

All mouse tissue microarray construction procedures were performed in the Stephenson Cancer Center-Cancer Tissue Pathology Core. For mouse neuroblastoma TMA, tumor tissues from non-metastatic xenograft-bearing animals and from multiple metastatic sites from high-risk aggressive disease-bearing animals were printed in duplicate. Immunohistochemical staining for GRB10, MYC, SEMA3D, KRTAP1-1, and MEGF10 was performed utilizing an automated Leica Bond III according to the manufacturer's protocol using the $B d^{\mathrm{m}}{ }^{\mathrm{m}}$ Polymer Refine detection system. Appropriate tissue morphologic/pathologic (H\&E) controls and negative controls with no primary antibody (data not shown) were examined in parallel. The slides were micro-digitally scanned using an Aperio Scanscope (Aperio Technologies, Inc., Buffalo Grove, IL, USA) slide scanner. This allows the assembly of tissue collections in TMA with variable magnifications. We constructed virtual slides with digital histology. The digital images of the TMA were then analyzed for GRB10, MYC, SEMA3D, KRTAP1-1 or MEGF10 specific positivity using the Aperio TMALab ${ }^{\text {tw }}$ software that is equipped with highly advanced algorithms for IHC and stain intensity including cytoplasmic-, nuclear-, membrane- and totalstaining Intensity and/or counts quantification. Automated staining positivity and intensity was quantified in precisely located and identified (with grid/row/column coordinates) individual cores within the TMA using protein specific (GRB10, SEMA3D, KRTAP1-1-cytoplasmic; MYC-nuclear; MEGF10-membranous) image analysis algorithms. Core-specific metadata for the TMA was exported to excel and the group-wise comparisons were performed with two-way ANOVA with Tukey's post-hoc correction (GraphPad Prism).

miRNA manipulations and quantitative highthroughput confocal immunofluorescence: To define the effect of characterized metastamiRs on the putative target proteins, we adopted two approaches: (i) inhibited hsa-miR-1224-3p or hsa-miR-1260 (both significantly upregulated) and (ii) functionally mimicked hsa-miR-125b, hsa-miR-27b, hsa-miR-93 or hsa-miR-20a (all significantly downregulated) and examined for the miRNA-dependent modulations in protein targets. Transient transfection of MSDACs with hsa-miR-125b-, hsa-miR-27b-, hsa-miR93- or hsa-miR-20a- mimics (MISSION ${ }^{\circ}$ microRNA Mimics, Sigma-Aldrich) as well as hsa-miR-1224-3p- and hsa-miR-1260-inhibitors (MISSION ${ }^{\circ}$ Synthetic miRNA Inhibitors, Sigma-Aldrich) were carried out by using either TurboFectin 8.0 reagent (Origene) or Neon electroporation transfection system (Life Technologies). We examined the cellular localization and expression levels of ADAMTS-1, ASK-1, CREB, FOSB, GRB10, EGFR, kRAS, MMP2, MMP3/10, p38, AKT, PTPN3, STAT3, TNF $\alpha$ and VEGFa in MSDACs using Operetta high content quantitative confocal imaging. Paraformaldehyde fixed cells were permeabilized ( $0.25 \%$ Triton X-100), blocked, and labelled with corresponding antibody. They were then tagged with Alexa Fluor 488 conjugated anti-mouse or anti-rabblit secondary antibody (Abcam). The nucleus was counterlabeled with DAPI. At least sixty-three fields/well with a minimum of $3 \mathrm{Z}$ planes were analyzed with integrated Columbus image analysis software. Unbiased automated batch analysis was performed and the cells-total number, mean/well cell-Alexa Fluor intensity were computed. Group-wise comparisons were performed with ANOVA with Tukey's post-hoc correction (GraphPad Prism).

\section{Clinical outcome association analysis}

We used the R2: microarray analysis and visualization platform (http://r2.amc.nl) created by Dr. Jan Koster at the Academic Medical Center (AMC), Amsterdam, to examine the association of the metastamiRs' key targets, i.e., those that showed defined alterations in protein expression/phosphorylation with immunoblotting, with overall survival of patients with neuroblastoma. This web-based application correlates a select gene expression profile with clinical outcomes using samples from various cohorts of patients, and permitted us to demonstrate the significance of altered genes in high-risk disease and their relevance to clinical outcomes. For this study, we utilized a cohort of 88 untreated primary human neuroblastoma samples and examined the prognostic values for ELK1, CDK4, CREB1, MMP2, AURK $\beta$, FRA, MYB, JUND, BIRC5, AKT2, SELE, TNF $\alpha$, NOS3, ESR1, KRTAP1-1, MMP3, NF2, CXCR4, ADAMTS1, 
CD54, EGFR, and AKT1 expression. Available clinical information on these patients include age group (<=18 m - 48; >18 m - 40), gender (Male 53, Female 35), INSS (Stage $1-8$, Stage $2-15$, Stage $3-13$, Stage 4 - 40, Stage 4S - 12), MYCN status (amplified 16, non-amplified 72), recurrence/progression (yes 35, not detected 53), alive (yes, 55, no 33) etc., (http://r2.amc.nl). The association of each gene's expression with the overall survival probability was plotted in Kaplan-Meier plots constructed for a follow-up of 240 months.

\section{Additional files}

Additional file 1: Figure S1. Ingenuity interaction networks for the miRNAs reorganized in aggressive high-risk metastatic neuroblastoma. All networks identified by IPA converge at biological functions that endorses metastatsis and, thereby signify their role as metastamiRs.

Additional file 2: Figure S2. Ingenuity pathway analysis: Heat map showing the association of the identified metastamiRs in multiple tumor systems. Box sizes correspond to the number of focus molecules and the color intensity is the - $\log (p$-value). Numerical labels in the boxes corresponds to the tumor type.

Additional file 3: Figure S3. Kaplan Meier plots showing clinical outcomes in a cohort of 88 neuroblastoma patients in association with the expression pattern of metastamiRs' protein targets, ELK1, CDK4, CREB1, MMP2, AURKB, FRA, MYB, JUND, BIRC5, AKT2, SELE, TNFa. All these targets showed induced expression levels (except CREB1) in MSDACs (compared to parental SH-SY5Y) and manifold of metastatic tumors (compared with non-metastatic xenograft) as examined with immunoblotting. Gene expression-associated clinical outcomes were assessed using the web-based R2: microarray analysis and visualization platform. Induced expression of ELK1, CDK4, MMP2, AURKB, FRA, MYB, JUND, BIRC5, AKT2, SELE and TNFa as well as decreased expression of CREB1 corresponded to the poor overall survival in NB patients.

Additional file 4: Figure S4. Kaplan Meier plots showing clinical outcomes in a cohort of 88 neuroblastoma patients in association with the expression pattern of metastamiRs' targets NOS3, ESR1, SELE, KRTAP1-1, MMP3, NF2, ELK-1, CXCR4, ADAMTS-1, ICAM-1, EGFR and ATK-1. All these targets showed induced expression levels in MSDACs (compared to parental SH-SY5Y) and manifold of metastatic tumors (compared with non-metastatic xenograft) as examined with immunoblotting.

Additional file 5: Table S1. List of reorganized miRNAs (52 up regulated and 22 down regulated), their defined (published evidence) functions in the context of metastasis and tumor progression, corresponding number of gene targets and the miRNA $\rightarrow$ metastatsis citation index. Almost all of the miRNAs identified in high-risk metastatic neuroblastomas has been well characterized in the perspective of metastasis regulation and, could be classified as metastamiRs.

Additional file 6: Reorganization of metastamiRs in the evolution of metastatic aggressive neuroblastoma cells.

\section{Abbreviations}

NB: Neuroblastoma; OS: Overall survival; EMT: Epithelial-mesenchymal transition; CSCs: Cancer stem cells; MSDACs: Metastatic sites derived aggressive cells; TMA: Tissue microarray.

\section{Competing interests}

The authors declare that they have no competing interests.

\section{Authors' contributions}

FHK - performed experiments; Data and statistical analysis; helped to draft the manuscript; VP - performed experiments; Data and statistical analysis; $S R$ - performed experiments; $S A$ - performed experiments; Data and statistical analysis; helped to drafted the manuscript; TSH - participated in the design of the study and contributed reagents. NA - conceived the study; participated in its design and coordination; performed experiments; Data and statistical analysis and; drafted the manuscript. We confirm that the manuscript has been read and approved by all named authors and that there are no other persons who satisfied the criteria for authorship but are not listed. We further confirm that the order of authors listed in the manuscript has been approved by all of us.

\section{Acknowledgements}

The authors are supported by the National Institutes of Health $(\mathrm{NIH}$ 1P20GM103639-01) from the COBRE Program of the National Institutes of Health Stephenson Cancer Center - Experimental Therapeutics Program Funds and OUHSC Department of Radiation Oncology Research Development Funds. The authors are thankful to the Stephenson Cancer Center (SCC) Cancer Tissue pathology core for all TMA and IHC services, SCC cancer functional genomics core for high-content confocal imaging, and OUHSC Flow Cytometry and Imaging Core for the cell sorting services. The authors also acknowledge the OUHSC Editorial Review of Scientific Communications (Ms. Kathy Kyler) for the help in critically reviewing this manuscript.

\section{Author details}

'Department of Radiation Oncology, University of Oklahoma Health Sciences Center, 940, Stanton L. Young Boulevard, BMSB 737, Oklahoma City, OK 73104, USA. ${ }^{2}$ Stephenson Cancer Center, 975 NE 10th Street, BRC 1468, Oklahoma City, OK 73104, USA.

Received: 18 February 2015 Accepted: 19 May 2015

Published online: 07 July 2015

\section{References}

1. Marc TG, Gurney. JG, Smith. MA, Olshan. AF: Sympathetic Nervous System Tumors. Cancer Incidence and Survival among Children and Adolescents: United States SEER Program 1975-1995, National Cancer Institute, Bethesda, MD 1999, NIH Pub. No. 99-4649(ICCC IV):65-72.

2. Gurney. JG, Smith. MA, Ross. JA: Cancer Among Infants. Cancer Incidence and Survival among Children and Adolescents: United States SEER Program 1975-1995, National Cancer Institute, Bethesda, MD 1999, NIH Pub. No. 99-4649 (XII):149-156

3. Morgenstern DA, Baruchel S, Irwin MS. Current and future strategies for relapsed neuroblastoma: challenges on the road to precision therapy. J Pediatr Hematol Oncol. 2013;35(5):337-47.

4. American-Cancer-Society:: Cancer Facts \& Figures Atlanta: American Cancer Society 2015:1-56

5. Smith MA, Seibel NL, Altekruse SF, Ries LA, Melbert DL, O'Leary M, et al. Outcomes for children and adolescents with cancer: challenges for the twenty-first century. J Clin Oncol. 2010;28(15):2625-34.

6. Maris JM, Hogarty MD, Bagatell R, Cohn SL. Neuroblastoma. Lancet. 2007;369(9579):2106-20.

7. Matthay KK, Reynolds CP, Seeger RC, Shimada H, Adkins ES, Haas-Kogan D, et al. Long-term results for children with high-risk neuroblastoma treated on a randomized trial of myeloablative therapy followed by 13-cis-retinoic acid: a children's oncology group study. J Clin Oncol. 2009;27(7):1007-13.

8. Cole KA, Maris JM. New strategies in refractory and recurrent neuroblastoma: translational opportunities to impact patient outcome. Clin Cancer Res. 2012;18(9):2423-8.

9. Santana VM, Furman WL, McGregor LM, Billups CA. Disease control intervals in high-risk neuroblastoma. Cancer. 2008;112(12):2796-801.

10. Simon T, Berthold F, Borkhardt A, Kremens B, De Carolis B, Hero B. Treatment and outcomes of patients with relapsed, high-risk neuroblastoma: results of German trials. Pediatr Blood Cancer. 2011;56(4):578-83.

11. Lau L, Tai D, Weitzman S, Grant R, Baruchel S, Malkin D. Factors influencing survival in children with recurrent neuroblastoma. J Pediatr Hematol Oncol. 2004;26(4):227-32.

12. Berthold F, Hero B, Breu H, Christiansen H, Erttmann R, Gnekow A, et al. The recurrence patterns of stages I, II and III neuroblastoma: experience with 77 relapsing patients. Ann Oncol. 1996;7(2):183-7.

13. Garaventa A, Parodi S, De Bernardi B, Dau D, Manzitti C, Conte M, et al. Outcome of children with neuroblastoma after progression or relapse A retrospective study of the Italian neuroblastoma registry. Eur J Cancer. 2009;45(16):2835-42. 
14. Weiss B, Vora A, Huberty J, Hawkins RA, Matthay KK. Secondary myelodysplastic syndrome and leukemia following 1311-metaiodobenzylguanidine therapy for relapsed neuroblastoma. J Pediatr Hematol Oncol. 2003;25(7):543-7.

15. London WB, Castel V, Monclair T, Ambros PF, Pearson AD, Cohn SL, et al. Clinical and biologic features predictive of survival after relapse of neuroblastoma: a report from the International Neuroblastoma Risk Group project. J Clin Oncol. 2011;29(24):3286-92.

16. Christofori G. New signals from the invasive front. Nature. 2006:441(7092):444-50.

17. Gupta GP, Massague J. Cancer metastasis: building a framework. Cell. 2006;127(4):679-95.

18. Schmidt-Kittler O, Ragg T, Daskalakis A, Granzow M, Ahr A, Blankenstein TJ, et al. From latent disseminated cells to overt metastasis: genetic analysis of systemic breast cancer progression. Proc Natl Acad Sci U S A. 2003;100(13):7737-42.

19. Kratimenos P, Koutroulis I, Marconi D, Syriopoulou V, DelivoriaPapadopoulos M, Chrousos GP, Theocharis S: Multi-targeted molecular therapeutic approach in aggressive neuroblastoma: the effect of Focal Adhesion Kinase-Src-Paxillin system. Expert opinion on therapeutic targets 2014;18(12):1395-406

20. Li Y, Li W, Zhang JG, Li HY, Li YM: Downregulation of tumor suppressor menin by miR-421 promotes proliferation and migration of neuroblastoma. Tumour biology. 2014;35(10):10011-7.

21. Morgenstern DA, London WB, Stephens D, Volchenboum SL, Hero B, Di Cataldo A, et al. Metastatic neuroblastoma confined to distant lymph nodes (stage $4 \mathrm{~N}$ ) predicts outcome in patients with stage 4 disease: A study from the International Neuroblastoma Risk Group Database. J Clin Oncol. 2014;32(12):1228-35.

22. Cheng L, Yang T, Kuang Y, Kong B, Yu S, Shu H, et al. MicroRNA-23a promotes neuroblastoma cell metastasis by targeting $\mathrm{CDH} 1$. Oncology letters. 2014;7(3):839-45.

23. Li Y, Wang H, Li J, Yue W. MiR-181C modulates the proliferation, migration, and invasion of neuroblastoma cells by targeting Smad7. Acta Biochim Biophys Sin. 2014;46(1):48-55

24. Hurst DR, Edmonds MD, Welch DR. Metastamir: the field of metastasisregulatory microRNA is spreading. Cancer Res. 2009;69(19):7495-8.

25. Bell E, Chen L, Liu T, Marshall GM, Lunec J, Tweddle DA. MYCN oncoprotein targets and their therapeutic potential. Cancer Lett. 2010;293(2):144-57.

26. Maris JM. Recent advances in neuroblastoma. N Engl J Med. 2010;362(23):2202-11.

27. Seeger RC, Brodeur GM, Sather H, Dalton A, Siegel SE, Wong KY, et al. Association of multiple copies of the $\mathrm{N}$-myc oncogene with rapid progression of neuroblastomas. N Engl J Med. 1985;313(18):1111-6.

28. Brodeur GM, Seeger RC, Schwab M, Varmus HE, Bishop JM. Amplification of $\mathrm{N}$-myc in untreated human neuroblastomas correlates with advanced disease stage. Science. 1984;224(4653):1121-4.

29. Brodeur GM. Neuroblastoma: biological insights into a clinical enigma. Nat Rev Cancer. 2003;3(3):203-16.

30. Brodeur GM, Bagatell R. Mechanisms of neuroblastoma regression. Nat Rev Clin Oncol. 2014;11(12):704-13.

31. Molenaar JJ, Koster J, Zwijnenburg DA, van Sluis $P$, Valentijn $L J$, van der Ploeg I, et al. Sequencing of neuroblastoma identifies chromothripsis and defects in neuritogenesis genes. Nature. 2012:483(7391):589-93.

32. Hogarty MD, Maris JM. PI3King on MYCN to improve neuroblastoma therapeutics. Cancer Cell. 2012;21(2):145-7.

33. Cohn SL, Tweddle DA. MYCN amplification remains prognostically strong 20 years after its "clinical debut". Eur J Cancer. 2004;40(18):2639-42.

34. Kawa K, Ohnuma N, Kaneko M, Yamamoto K, Etoh T, Mugishima H, et al. Long-term survivors of advanced neuroblastoma with MYCN amplification: A report of 19 patients surviving disease-free for more than 66 months. J Clin Oncol. 1999;17(10):3216-20

35. Cohn SL, London WB, Huang D, Katzenstein HM, Salwen HR, Reinhart T, et al. MYCN expression is not prognostic of adverse outcome in advanced-stage neuroblastoma with nonamplified MYCN. J Clin Oncol. 2000;18(21):3604-13.

36. Bartel DP. MicroRNAs: genomics, biogenesis, mechanism, and function. Cell. 2004;116(2):281-97.

37. Bottai G, Pasculli B, Calin GA, Santarpia L: Targeting the microRNA-regulating DNA damage/repair pathways in cancer. Expert opin biol ther 2014;14(11):1667-83

38. Chen Y, Liu XR, Yin YQ, Lee CJ, Wang FT, Liu HQ, et al. Unravelling the multifaceted roles of Atg proteins to improve cancer therapy. Cell Prolif. 2014;47(2):105-12

39. Fu J, Xu X, Kang L, Zhou L, Wang S, Lu J, et al. Biochem Biophys Res Commun. 2014:445(2):314-9.
40. Elzein S, Goodyer CG. Regulation of Human Growth Hormone Receptor Expression by MicroRNAs. Mol Endocrinol. 2014;28(9):1448-59.

41. Liu F, Lv Q, Du WW, Li H, Yang X, Liu D, et al. Specificity of miR-378a-5p targeting rodent fibronectin. Biochim Biophys Acta. 2013;1833(12):3272-85.

42. Kim VN. MicroRNA biogenesis: coordinated cropping and dicing. Nat Rev Mol Cell Biol. 2005;6(5):376-85.

43. Cheung IY, Farazi TA, Ostrovnaya I, Xu H, Tran H, Mihailovic A, et al. Deep MicroRNA sequencing reveals downregulation of miR-29a in neuroblastoma central nervous system metastasis. Genes Chromosomes Cancer. 2014;53(10):803-14.

44. Gonzalez-Nunez V, Noriega-Prieto JA, Rodriguez RE. Morphine modulates cell proliferation through mir133b \&mir128 in the neuroblastoma SH-SY5Y cell line. Biochim Biophys Acta. 2014;1842(4):566-72.

45. Chen X, Pan M, Han L, Lu H, Hao X, Dong Q. miR-338-3p suppresses neuroblastoma proliferation, invasion and migration through targeting PREX2a. FEBS Lett. 2013;587(22):3729-37.

46. Qiao J, Lee S, Paul P, Theiss L, Tiao J, Qiao L, et al. miR-335 and miR-363 regulation of neuroblastoma tumorigenesis and metastasis. Surgery. 2013;154(2):226-33

47. Ye J, Wu X, Wu D, Wu P, Ni C, Zhang Z, et al. miRNA-27b targets vascular endothelial growth factor $C$ to inhibit tumor progression and angiogenesis in colorectal cancer. PLoS One. 2013;8(4):e60687.

48. Zhang H, Pu J, Qi T, Qi M, Yang C, Li S, et al. MicroRNA-145 inhibits the growth, invasion, metastasis and angiogenesis of neuroblastoma cells through targeting hypoxia-inducible factor 2 alpha. Oncogene. 2014;33(3):387-97.

49. Xin $C$, Buhe $B$, Hongting $L$, Chuanmin $Y$, Xiwei $H$, Hong $Z$, et al. MicroRNA-15a promotes neuroblastoma migration by targeting reversion-inducing cysteine-rich protein with Kazal motifs (RECK) and regulating matrix metalloproteinase-9 expression. FEBS J. 2013:280(3):855-66.

50. Zhang H, Qi M, Li S, Qi T, Mei H, Huang K, et al. microRNA-9 targets matrix metalloproteinase 14 to inhibit invasion, metastasis, and angiogenesis of neuroblastoma cells. Mol Cancer Ther. 2012;11(7):1454-66.

51. Meseguer S, Mudduluru G, Escamilla JM, Allgayer H, Barettino D. MicroRNAs10a and -10b contribute to retinoic acid-induced differentiation of neuroblastoma cells and target the alternative splicing regulatory factor SFRS1 (SF2/ASF). J Biol Chem. 2011;286(6):4150-64

52. Kovalevich J, Langford D. Considerations for the use of SH-SY5Y neuroblastoma cells in neurobiology. Methods Mol Biol. 2013;1078:9-21.

53. Aravindan S, Natarajan M, Awasthi V, Herman TS, Aravindan N. Novel synthetic monoketone transmute radiation-triggered NFkappaBdependent TNFalpha cross-signaling feedback maintained NFkappaB and favors neuroblastoma regression. PLoS One. 2013;8(8):e72464.

54. Veeraraghavan J, Natarajan M, Aravindan S, Herman TS, Aravindan N. Radiation-triggered tumor necrosis factor (TNF) alpha-NFkappaB crosssignaling favors survival advantage in human neuroblastoma cells. J Biol Chem. 2011;286(24):21588-600.

55. Chen X, Zhu Y, Han L, Lu H, Hao X, Dong Q. Chemokine receptor 4 gene silencing blocks neuroblastoma metastasis in vitro. Neural Regeneration Res. 2014;9(10):1063-7.

56. Tian X, Hou W, Bai S, Fan J, Tong H, Xu H. XAV939 inhibits the stemness and migration of neuroblastoma cancer stem cells via repression of tankyrase 1. Int J Oncol. 2014:45(1):121-8.

57. Feduska JM, Aller SG, Garcia PL, Cramer SL, Council LN, van Waardenburg RC, Yoon KJ: ICAM-2 confers a non-metastatic phenotype in neuroblastoma cells by interaction with alpha-actinin. Oncogene 2015:34(12):1553-62.

58. Schwankhaus N, Gathmann C, Wicklein D, Riecken K, Schumacher U, Valentiner U: Cell adhesion molecules in metastatic neuroblastoma models. Clinical \& experimental metastasis 2014;31(4):483-496.

59. Guo J, Dong Q, Fang Z, Chen X, Lu H, Wang K, et al. Identification of miRNAs that are associated with tumor metastasis in neuroblastoma. Cancer Biol Ther. 2010;9(6):446-52.

60. Almeida Ml, Reis RM, Calin GA. MicroRNAs and metastases-the neuroblastoma link. Cancer Biol Ther. 2010;9(6):453-4

61. Khew-Goodall Y, Goodall GJ. Myc-modulated miR-9 makes more metastases. Nat Cell Biol. 2010;12(3):209-11.

62. Natarajan M, Aravindan N, Meltz ML, Herman TS. Post-translational modification of I-kappa B alpha activates NF-kappa B in human monocytes exposed to $56 \mathrm{Fe}$ ions. Radiat Environ Biophys. 2002:41(2):139-44. 